\title{
COVID-19 and Sleep in Medical Staff: Reflections, Clinical Evidences, and Perspectives
}

\section{Luigi Ferini-Strambi, MD, PhD ${ }^{1,2, *}$}

Marco Zucconi, $M D^{2}$

Francesca Casoni, $M D^{2}$

Maria Salsone, $M D^{2,3}$

\author{
Address \\ ${ }^{1}$ Vita-Salute San Raffaele University, Milan, Italy \\ ${ }^{*}, 2$ Sleep Disorders Center, Division of Neuroscience, IRCCS San Raffaele Hospital, \\ Milan, Italy \\ Email: ferinistrambi.luigi@hsr.it \\ ${ }^{3}$ Institute of Molecular Bioimaging and Physiology, National Research Council, \\ Catanzaro, Italy
}

Published online: 6 August 2020

(C) Springer Science+Business Media, LLC, part of Springer Nature 2020

This article is part of the Topical Collection on Reflections from the COVID Pandemic

Keywords COVID-19 pandemic - Sleep quality · Actigraphy · Healthcare workers/medical staff

\section{Abstract}

Purpose of the review There is evidence that, before the coronavirus pandemic 2019 (COVID-19), healthcare workers did not experience good sleep quality with relevant consequences on health. By contrast, little is known about the sleep quality of medical staff during the COVID-19 pandemic. In this review, we aimed to contribute with a review of the literature, sharing our clinical experience supported by actigraphic evaluation and by proposing future strategies.

Recent findings Sleep disorders, in particular insomnia, have been commonly reported in frontline medical workers, in hospitals during the COVID-19 pandemic and are often accompanied by depressive and anxiety symptoms. Sleep quality, however, has been mainly assessed by the use of self-reported measures, thus limiting clinical usefulness. Summary Poor sleep quality among the medical staff is prevalent, and our experience supports that this has increased during the COVID-19 pandemic. A longitudinal investigation assessing whether and for how long sleep remains altered in medical staff could be of interest to evaluate the temporal effect of the pandemic on health. 


\section{The state of the art}

In the weeks of May 2020, during which this report was written, the COVID-19 pandemic in Lombardia, Italy, was subsiding and likely entering a novel phase. Starting February 20, the day on which the first case of SARSCov-2 infection-related severe acute respiratory syndrome (COVID-19) was diagnosed in an Italian citizen in Codogno, the pandemic has had severe and profound effects on all aspects of our life including mental and physical health. Considering the clinical relevance that good sleep quality has on our health even perhaps importantly among healthcare workers, we would like to contribute with an accurate review of the literature, sharing our clinical experience and proposing future strategies.

The COVID-19 pandemic raises some fundamental questions: (1) what was the impact of COVID-19 infection on the sleep quality of medical staff, and (2) what are the effects of impaired sleep on the mental and physical health of medical staff? Although the COVID19 pandemic is described as changing the world, in some cases, it has likely accentuated a pre-existing trend. Thus, it is of relevance to consider sleep conditions in healthcare workers before the COVID-19 pandemic. Some studies have evaluated sleep quality and its relationship to cognitive factors among healthcare workers, in particular nurses. According to the findings of a recent meta-analysis [1] examining the pooled prevalence of sleep quality across fifty-three studies, poor sleep quality was a very common feature and was reported in $61.0 \%$ of nursing staff. This was characterized by alterations of sleep parameters such as sleep latency, duration, and sleep efficiency (SE), subjective sleep quality, and daytime dysfunctions. Moreover, subgroup and metaregression analyses found that Pittsburgh Sleep Quality Index (PSQI) cutoff values, mean age, body mass index, sample size, study quality, and work experience impacted the prevalence of poor sleep quality [1]. These authors concluded that effective measures should be taken to improve poor sleep quality in this critical population given its negative impact on health. Indeed, lack of sufficient sleep can create numerous problems for healthcare workers, and particular attention to the role of cognitive and metacognitive processes is due in order to improve the sleep quality [2]. Finally, poor sleep quality is highly prevalent not only among healthcare workers but also among students, and early detection and management is also recommended [3].
What changed during the COVID-19 pandemic? Due to the short time since the pandemic was first recognized, there is scant literature on its impact on sleep quality in healthcare workers. The study by Ran et al. represents an important starting point $[4 \bullet \bullet]$. This retrospective cohort study in a Hospital of Wuhan, China, demonstrated that high-risk department (e.g., those in which interventional medical or surgical procedures that generate respiratory aerosols are performed), longer duty hours, and suboptimal hand hygiene are all potential

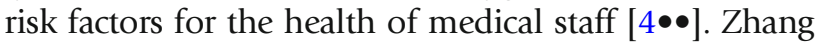
et al. were among the first to investigate the prevalence of sleep disorders, in particular insomnia among a total of 1563 medical staff members including frontline medical workers, in hospitals during the COVID-19 outbreak $[5 \bullet \bullet$. Their main results demonstrated that during this period, more than one-third of the medical staff suffered insomnia symptoms according to the Insomnia Severity Index (total score $\geq 8$ ) accompanied by depressive and anxiety symptoms. Of note, in their cohort, the insomnia symptoms were also associated with education level, work in an isolation unit, concern about being infected, perceived lack of helpfulness in terms of psychological support from social media, and very strong uncertainty

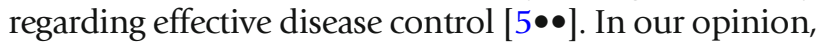
this study represents a call to consider the sociopsychological factors as potential risk factors for insomnia among medical staff and to intervene with personalized treatment such as cognitive insomnia behavior therapy. In accordance with this, Berligieri et al. have subsequently proposed to not only limit analyses to the demographic characteristics of workers and the presence of protective equipment but also to consider working conditions in the hospital, including an analysis of shift work and its effects of sleep quality [6]. Finally, in a very recent cross-sectional study, Wang et al. also assessed the effect of the COVID-19 outbreak on the sleep quality of 123 healthcare workers in a children's healthcare center in Wuhan [7•]. Their analysis consisted of questionnaire completed by participants on their own smartphone and was divided in three parts, including sociodemographic characteristics and COVID-19 epidemic-related factors, the PSQI, and Zung's self-rating anxiety scale and self-rating depression scale. During the COVID-19 outbreak, about $38 \%$ of pediatric healthcare workers were suffering from sleep disturbance and about $20 \%$ had anxiety and depression [7•]. A trend indicative of a higher prevalence of sleep disturbances 
among doctors rather than nurses was also noted although the difference was not statistically significant.

In summary, these early studies suggest that healthcare workers and students do not experience good quality of sleep and that this has increased during the COVID-19 pandemic. Several variables and potential risk factors, such as education level or work in an isolation unit, appear to play a crucial role in moderating the sleep disturbance. These evaluations of sleep quality in medical staff, however, have mainly being assessed using qualitative and self-reporting measures such as sleep questionnaire, thus limiting clinical usefulness.

\section{Clinical evidences: our experience}

In order to provide a complete narrative description during the COVID-19 outbreak, we report on our clinical experience at the Sleep Disorder Center of the IRCCS San Raffaele Scientific Institute, Milan. Here, we carried out the first actigraphic study investigating the prevalence of sleep disorders among healthcare workers during the pandemic. Data were consecutively collected from 40 medical staff that treated patients with COVID-19 infection. Study participants were either doctors or nurses working in the Departments of Infectious Disease, Respiratory Medicine, or the Intensive Care Unit of San Raffaele Hospital. In this observational study, we used a combination of actigraphy and validated clinical questionnaires to quantitatively and quantitatively assess the sleep quality among the medical staff. Of interest, before the COVID-19 pandemic, our participants did not suffer sleep disorders including insomnia and did not take pharmacological treatments. In our cohort, we observed a gender prevalence as about $70 \%$ of the participants were females, mainly nurses, with an age range from 26 to 50 years. Our analysis revealed that about $35 \%$ of healthcare workers were suffering from sleep disturbances having a SE value less than $90 \%$ and PSQI scores ranging from 7 to 21 (Global PSQI score : 0-21; PSQI abnormal score > 5) [8]. We also found a significant negative correlation between SE and PSQI $(r=-0.54 ; p=0.04)$ and a trend of a negative association between SE and age. These preliminary findings are in line with the previous reports $[5,7]$ and demonstrate that healthcare workers had poor sleep quality while caring for COVID-19 patients. A longitudinal investigation assessing whether and for how long sleep remains altered in medical staff could be of interest to evaluate the temporal effect of the pandemic on health.

In our opinion, it is noteworthy to investigate sleep quality among the medical staff for several reasons. First, there is the possibility that poor sleep can significantly modify the susceptibility to COVID-19 infection. Indeed, it is well-documented that good sleep quality and normal function of the immune system are strongly related. In this context, Meira e Cruz et al. have recently reviewed the potential links between SARS-Cov- 2 infection, circadian rhythms, and sleep [9]. Although it is unclear how disrupted or insufficient sleep may affect SARS-Cov2 infection rates and the severity of its clinical manifestations, these authors suggested that the immuno-inflammatory activation that typically occurs in lung injury induced by COVID-19 may be influenced by the circadian clock [9]. It is speculative that deregulation of circadian rhythms such as that occurs in night shift workers or with jetlag could play a disease-specific role by altering the susceptibility to infection or modifying the clinical manifestations of COVID-19 [9]. In addition, it has also been reported that 
angiotensin II (ATII) affects the rhythmic expression of key repressors that constitute the feedback loop of mammalian clock circuitry in the suprachiasmatic nuclei $[9,10]$. These studies open new scenarios for understanding the mechanisms underlying this interesting relationship. Secondly, cumulating evidence supports the implications of disrupted sleep during the COVID-19 pandemic on mental health of medical staff. Hang $\mathrm{Y}$ et al. collected data from 7236 self-selected volunteers including healthcare workers to assess the mental health of the Chinese public during the outbreak and to explore potential influencing factors. Healthcare workers were more likely to have poor sleep quality and to be at higher risk of mental illness including generalized anxiety disorder and depressive symptoms [11]. In light with these reports, a crosssectional observational study including 180 medical staff who treated patients with COVID-19 in January and February 2020 in Wuhan revealed that healthcare workers had increased levels of anxiety, stress, and self-efficacy that were dependent on sleep quality and social support [12]. Finally, a recent comment in Lancet Psychiatry appears as a call for action to protect the mental health of medical staff in order to better control the epidemic as well as their own long-term health [13]. Overall, these findings demonstrate that alterations of circadian rhythms and disturbed sleep could increase the susceptibility of medical staff to COVID-19 infection.

\section{Perspectives and future strategies}

\section{Conclusions}

This is the time for teamwork in order to elaborate common future strategies to mitigate poor sleep quality and its associated risks. Achieving this is particularly relevant to the medical community since the psychological impact of the COVID-19 pandemic is likely to persist for a long time after the end of an epidemic, as occurred for the SARS epidemic in 2003 [13].

Thus, the implementation of healthy sleep measures as a potential protective strategy against COVID-19 infection should be encouraged. As a first possible intervention, most general hospitals in Wuhan, China, have established a shift system to allow front-line medical workers to rest and to take turns in high-pressured roles. Second, according to the data from Kang et al., medical workers who received psychological interventions by both a medical (mainly psychiatric) team and assistance from telephone hotlines

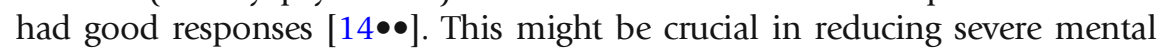
stress and thus improve sleep quality among medical staff. Lastly, there is an urgent need for research to better understand the neurobiological mechanisms linking the effects of the COVID-19 pandemic to disrupted sleep and vice versa, how disrupted sleep may lead to high susceptibility to COVID-19, and clinical manifestations of the disease. These studies will require multidisciplinary teams and integration across several disciplines and sectors.

In summary, the time is mature for some reflections regarding the COVID-19 pandemic and its effects (direct and indirect) on healthcare workers. First, healthcare workers usually do not experience good quality of sleep, and this has been exacerbated by the COVID-19 pandemic as confirmed by the clinical 
experience at our Sleep Disorder Center. Second, considering the strict relationship between sleep disorders and cognitive and mental disorders, as well as the clinical implications for mental health, emphasis should be placed on the early detection of sleep disorders in healthcare personal caring for patients with COVID-19. This information will be applicable to unexpected infections/ pandemics in the future, allowing for optimal responses.

\section{References and Recommended Reading}

Papers of particular interest, published recently, have been highlighted as:

- Of importance

• Of major importance

1. Zeng LN, Yang Y, Wang C, Li XH, Xiang YF, Hall B. Prevalence of poor sleep quality in nursing staff: a meta-analysis of observational studies. Behav Sleep Med. 2019;31:1-14. https://doi.org/10.1080/ 15402002.2019.1677233.

2. Khatony A, Zakiei A, Khazaie H, Rezaei M, Janatolmakan M. International nursing: a study of sleep quality among nurses and its correlation with cognitive factors. Nurs Adm Q. 2020;44(1):E1-E10. https://doi.org/10.1097/NAQ.0000000000000397.

3. Belingheri M, Pellegrini A, Facchetti R, De Vito G, Cesana G, Riva MA. Self-reported prevalence of sleep disorders among medical and nursing students. Occup Med (Lond). 2020;70(2):127-30. https://doi.org/10. 1093/occmed/kqaa011.

4.• Ran L, Chen X, Wang Y, Wu W, Zhang L, Tan X. Risk factors of healthcare workers with coronavirus disease 2019: a retrospective cohort study in a designated Hospital of Wuhan in China. Clin Infect Dis. 2020. https://doi.org/10.1093/cid/ciaa287.

This study showed the importance of an early identification of potential risk factors for the health of medical staff.

5.•• Zhang C, Yang L, Liu S, Ma S, Wang Y, Cai Z, et al. Survey of insomnia and related social psychological factors among medical staff involved in the 2019 novel coronavirus disease outbreak. Front Psychiatry. 2020;11:306. https://doi.org/10. 3389/fpsyt.2020.00306 eCollection 2020.

This is the first study investigating the prevalence of sleep disorders among frontline medical workers, in hospitals during the COVID-19 outbreak.

6. Belingheri M, Paladino ME, Riva MA. Working schedule, sleep quality and susceptibility to COVID-19 in healthcare workers. Clin Infect Dis. 2020. https://doi. org/10.1093/cid/ciaa499.
7.• Wang S, Xie L, Xu Y, Yu S, Yao B, Xiang D. Sleep disturbances among medical workers during the outbreak of COVID-2019. Occup Med (Lond). 2020. https://doi.org/10.1093/occmed/kqaa074 This study is focused on the identification of sleep disturbance, anxiety and depression among the medical staff.

8. Curcio C, Tempesta D, Scarlata S, Marzano C, Moroni F, Rossini PM. Validation of the Italian version of Pittsburgh Sleep Quality Index (PSQI). Neurol Sci. 2013;34:511-9.

9. Meira e Cruz M, Miyazawa M, Gozal D. Putative contributions of circadian clock and sleep in the context of SARS-CoV-2 infection. Eur Respir J. 2020; in press. https://doi.org/10.1183/13993003.01023-2020.

10. Li W, Zhang C, Sui J, Kuhn JH, Moore MJ, Luo S, et al. Receptor and viral determinants of SARS-coronavirus adaptation to human ACE2. EMBO J. 2005;24(8):1634-43.

11. Huang Y, Zhao N. Mental health burden for the public affected by the COVID-19 outbreak in China: who will be the high-risk group? Health med. 2020 14:1-12. doi: https://doi.org/10.1080/13548506.2020. 1754438.

12. Xiao H, Zhang Y, Kong D, Li S, Yang N. The effects of social support on sleep quality of medical staff treating patients with coronavirus disease 2019 (COVID-19) in January and February 2020 in China. Med Sci Monit. 2020;26:e923549. https://doi.org/10.12659/MSM. 923549.

13. Maunder RG, Lancee WL, Balderson KE, Bennett JP, Borgundvaag B, Evans S, et al. Long-term psychological and occupational effects of providing hospital healthcare during SARS outbreak. Emerg Infect Dis. 2006;12(12):1924-32. 
14.• Kang L, Li Y, Hu S, Chen M, Yang C, Xiang Yang B, et al The mental health of medical workers in Wuhan, China dealing with the 2019 novel coronavirus. Lancet

Psychiatry. 2020;7(3):e14. https://doi.org/10.1016/ S2215-0366(20)30047-X

This article showed the importance to protect the mental health of medical staff.

\section{Publisher's Note}

Springer Nature remains neutral with regard to jurisdictional claims in published maps and institutional affiliations. 